\title{
Bacterial Cells as Model Factories
}

\author{
Ichiro Matsumura \\ Department of Biochemistry, Center for Fundamental and Applied Molecular Evolution, Emory University, Atlanta, USA \\ Email: imatsum@emory.edu
}

Received September 12, 2012; revised October 14, 2012; accepted October 30, 2012

\begin{abstract}
Bacteria, like industrial engineers, must manage processes that convert low value inputs into high value outputs. Bacteria are not intelligent, so they utilize self-organizing production systems to accelerate life-sustaining chemical processes. Here I explore two questions. First, can businesses apply the principles of self-organization? Second, can operations researchers contribute to our understanding of biological systems? I explain biochemical concepts in plain terms, illustrated with a few informative laboratory evolution experiments, and describe the organizing principles that underlie complex biological systems. I describe the new disciplines of synthetic biology and metabolic engineering, which offer opportunities for interdisciplinary collaboration between life scientists and operations researchers.
\end{abstract}

Keywords: Complex Systems; Control Theory; Synthetic Biology; Metabolic Engineering

\section{Cells Are Self-Organizing Factories}

Imagine the perfect factory, one that manufactures high value products quickly and efficiently according to demand, resists disruptions, but readily reorganizes to produce new products in response to new market opportunities. Bacterial cells evince these properties, although the analogy is imperfect. Bacteria don't require human employees who are sometimes driven by motivations that don't align with organizational goals. Furthermore, bacteria are "selfish", as they evolved to produce compounds for themselves rather than for sometimes fickle human customers. The similarities and differences between factories and bacteria are nevertheless informative, and offer lessons to industrial engineers and biologists alike.

Bacteria face challenges analogous to those that confront factory managers. They must convert low value inputs (substrates, such as sunlight, water or carbon dioxide) into higher value outputs (products, such as sugar) to survive. All organisms are obliged to compete for food, so their life-sustaining processes must be fast (high throughput or flux), resource efficient (lean processes, with minimal bottlenecks and waste products) and precise (low process variation). Bacteria live in changing environments, so they must detect molecular signals, withstand health threats and make efficient process tradeoffs (appropriate prioritization of cost, quality, time, flexibility) in accordance with their needs. Bacteria are not intelligent, and unlike factories are self-assembling, self-regulating, self-repairing and self-replicating. Most importantly, they invent new systems that evolve without guidance from any intelligent designer. Indeed, an under- standing of adaptive molecular evolution is the key to understanding how and why the manufacturing operations of a cell self-organize.

The purpose of this review is to pose two questions. First, can operations researchers design self-organizing businesses? Second, can mathematical modeling be used to understand and improve biological systems? Solutions to these problems could be theoretically important and economically valuable, but will not be easy. They will likely require interdisciplinary collaboration that will be impeded by the linguistic barriers between the biological and industrial sciences. 1 will first review some fundamental biochemical concepts in lay terms (Section 2). Readers already well-versed in the scientific jargon of enzymes and metabolism are welcomed to skip ahead to Section 3, which describes the nature of complex systems. Lessons for operations researchers are summarized in Section 4, and collaborative opportunities with synthetic biologists and metabolic engineers are described in Section 5.

\section{Biochemical Concepts in Plain English}

All living things (organisms) survive by converting nutrients into chemical energy (catabolism), and using that energy to convert available building block molecules into cellular infrastructure (anabolism). To a biochemist, this set of self-sustaining processes IS life. Most of these reactions do not occur rapidly enough under physiological conditions (in water at neutral $\mathrm{pH}$ at body temperature) to support life, so cells create tiny protein machines called enzymes that accelerate the conversion of input (substrate 
molecules) into output (product molecules). Each enzyme catalyzes at least one assembly process (chemical reaction), and is thus analogous to a piece of manufacturing equipment within a factory.

Enzymes, like modern manufacturing equipment, are incredibly fast and precise. Many have maximum capacities (or turnover numbers) of thousands of chemical conversion events per second; some catalyze millions of events per second. Enzymes typically cause reactions to occur a billion times faster than they otherwise would [1] — a reaction that would normally take 30 years to complete in a test tube would occur within a second after the addition of enzyme. It has to be the right enzyme though, as most are highly specific, which is to say that most are thought to recognize a particular substrate and convert it into a particular product. Recent experimental results, including some from my laboratory, suggest that enzymes are not perfectly precise. I will explain the implications of these findings below.

Individual enzymes are not sufficient to transform sugar into chemical energy, or to convert sunlight, water and carbon dioxide into sugar and oxygen. Enzymes work in assembly lines called metabolic pathways, in which the product of one enzyme becomes the substrate for the next. Each pathway is a collection of enzymes that carries out a process, defined as "collection of tasks, connected by flows of goods and information, that transforms various inputs into more valuable outputs" [2]. Most of the substrates and products within a cell at any given time are metabolic intermediates, analogous to unfinished inventory within the continuous processes of an operational factory. For example, the pathway that produces cholesterol, a valuable compound essential for cell membranes and steroid hormones, from acetyl-CoA, the common breakdown product of carbohydrates, lipids and proteins, includes 12 enzyme-catalyzed reactions. Nearly all steps are thermodynamically unfavorable, which means that they are very unlikely to occur in the absence of a catalyst. In the cell they are coupled by the enzymes to the expenditure of chemical energy extracted (by other enzymes) during the breakdown of nutrients into acetyl-CoA. The human body thus produces cholesterol whenever it is absent from the diet.

All of the metabolic pathways in a cell connect through their substrates and products to form a single metabolic network. Maps of these networks resemble the process flow diagrams for the manufacture of complicated products. Only a subset of the metabolic pathways are activate at any given time, in accordance with the needs of the cell. The production and utilization of chemical energy in a cell is analogous to the cash flows of a business. Anabolic pathways, such as those that build the biological infrastructure of the cell, require energy inputs that are analogous to operational expenses. Over the long run, the catabolic (energy producing) pathways must pro- duce at least as much energy as that utilized by anabolic pathways, so that the cell can achieve balance (steadystate).

Bacteria, like businesses, do not live in constant environments except perhaps in the protected confines of a laboratory. Metabolic pathways are regulated through a variety of mechanisms. Enzymes depreciate (unfold) quickly for reasons explained below, so they can be regulated by increasing or decreasing the rates at which they are replaced. Some enzymes, generally those that catalyze the rate-determining step (bottleneck), are activated or deactivated by the substrates or products of other enzymes. This transistor-like form of control, called allostery, enables almost instantaneous feedback regulation of pathways. Some enzymes catalyze the chemical modification of other enzymes-indeed multi-enzyme signaling pathways mediate most of the information flow from the exterior a cell to the enzymes within. These regulatory mechanisms collectively enable appropriate responses to changes in intracellular inventory counts and the external environment.

For example, the enzyme 3-hydroxy-3-methyl-glutaryl-CoA reductase (HMG-CoA reductase), which catalyzes the rate-limiting step of the aforementioned biosynthesis of cholesterol, is regulated in several ways. The logic is straightforward-HMG-CoA reductase is activated whenever chemical energy is available-but can seem complicated in the obscure jargon of metabolic biochemistry. The Sterol Regulatory Element Binding Protein activates the transcription of the HMB-CoA Reductase gene when cholesterol levels are low. When the concentration of Adenosine Mono Phosphate is high, indicating low levels of chemical energy (since AMP is a precursor to ATP, the chemical energy currency of the cell), a regulatory enzyme called AMP-activated protein kinase inactivates HMG-CoA reductase by catalyzing the attachment of a phosphate group. Another regulatory enzyme, HMG-CoA reductase phosphatase, which is itself activated by the hormone insulin through a complex multi-protein mechanism, catalyzes the removal of that phosphate group, and the consequent re-activation of HMG-CoA reductase [3]. This key enzyme is regulated in at least two other ways. The logic is simple but the biochemical mechanisms are too complicated to discuss here. Incidentally, these regulatory mechanisms apparently evolved in a low cholesterol world. Too much cholesterol can cause heart disease, so many people today must reduce their dietary intake and artificially inhibit HMG-CoA reductase with statins, such as Lipitor ${ }^{\mathrm{TM}}$, Crestor $^{\mathrm{TM}}$ and Zocor $^{\mathrm{TM}}$.

\section{Enzymes, Metabolic Pathways and Regulatory Networks Are Complex Systems}

All biological systems are complex, which is to say that 
their emergent properties are functions of interactions between their components. Enzymes are proteins, which are chains of subunits (amino acids) that fold up (selfassemble) into three-dimensional shapes. All proteins are composed of the same 20 amino acids, just as all English words are composed of some subset of the same 26 letters. Proteins with different amino acid sequences fold up into different three dimensional shapes with different functions. Biological complex systems are holons, which means that they function as components are larger complex systems [4]. Metabolic pathways are composed of enzymes. Metabolic networks are composed of pathways. Populations are composed of individual organisms. Ecosystems are composed of populations. Businesses are similarly complex at each level of order. It is therefore impractical to formulate models, of either metabolic networks or businesses, without making some simplifying assumptions.

All complex systems evolve, and this property enables self-assembly, self-repair and self-replication. The protein sequences are encoded by DNA (deoxyribonucleic acid) sequences, which are subject to change through mutation. The three-dimensional fold (conformation) of a wild-type (non-mutant) protein is critical for function, but is also fragile. Relatively modest changes in temperature or concentration can cause a protein to unfold, aggregate and lose its biological function, which incidentally is why normally transparent egg "whites" solidify and turn white when they are cooked. Deleterious mutations can cause DNA to encode novel protein sequences that never fold properly, and therefore never function at all. Beneficial mutations, which are much rarer, lead to improvements in the function (speed, specificity) or durability (stability) of a protein, thereby imparting a competitive advantage upon the whole organism.

My research team, and many others, studies the adaptive evolution of proteins in the laboratory. Random mutations can be introduced into protein-coding genes as they are amplified (under conditions that compromise the fidelity of the enzyme that copies DNA $[5,6])$. The mutated genes are inserted into a "vector", most commonly a circular "plasmid" DNA that is replicated by the enzymes within bacteria, which are then injected into live cells. The "transformed" cells, those now carrying a mutant gene and plasmid, are induced to express (produce) mutant protein and evaluated in high throughput screens. Cells, or colonies of genetically identical cells, that exhibit properties (such as reactivity with a substrate that changes color in reactions with enzyme) are isolated and characterized. Multiple rounds of random mutation and screening can lead to the evolution of protein variants quite different from their ancestors [7]. The lessons that we learn from these "directed evolution" experiments, and from the subsequent biochemical analysis of the evolved proteins, and the intermediate forms, are described below.

Charles Darwin understood 150 years ago that variation, which we now know is caused by mutation, and natural selection can improve the fitness of a biological system. It was not obvious then how new functions originate. Theologian William Paley captured the dilemma well when he wrote "The existence of a watch implies the existence of a watchmaker" [8]. Contemporary Creationists still wonder out loud how complex systems can originate by chance if their components cannot function on their own. Darwin's responded by hypothesizing that biological parts that were selected for one function could nevertheless end up with other "promiscuous" activities [9]. Darwin's hypothesis has received ample experimental support [10], and we and others have demonstrated that it holds true at the molecular level.

Wayne Patrick, now a faculty member at Otago University in New Zealand, screened the genes of the bacterium Escherichia coli. He found over a dozen that were multifunctional, each with secondary activity strong enough to rescue cells from starvation [11]. The screen was limited to a small subset (2.4\%) of the genes (each with at least one function) within this bacterium so we predict that multi-functionality is common among enzymes [12]. Our results suggest that many functions are redundant, which make E. coli, and presumably all other organisms, more robust (resilient) against mutations. In combination with the directed evolution experiments reviewed above, they also suggest a plausible mechanism for the evolution of novel catalytic functions. Suppose that a population of bacteria were challenged with a new toxin, or a new potential nutrient. The multifunctional proteins with those bacteria could potentially serve as seeds for the next steps in evolutionary innovation, just as redundant manufacturing equipment could be re-purposed for the production of a new product. The modularity of DNA and protein sequences enables adaptation to changes in the chemical environment, just as the design principles of service oriented architectures help businesses adapt to changes in the marketplace.

\section{Lessons for Operations Researchers}

Biologists have used the bacterium, E. coli, as a model system (or reference organism) for over a century [13]. They continue to study the structure and function of its metabolic network, but the lessons learned so far could be instructive to operations researchers. As I argued above, bacteria rely not upon intelligence but rather upon systems that are self-assembling, self-repairing and selfreplicating to convert low value inputs into high value outputs. The Darwinian formula of genetic variation and natural selection enables self-organization. We and many other have shown that laboratory evolution can increase 
the stability and/or catalytic efficiency of enzymes (vide supra). An analogous selection process has improved manufacturing productivity throughout the global economy since the start of the Industrial Revolution. The directed evolution of factories is not practical as a design tool, but computer simulations of competing virtual businesses could well be informative.

My co-workers and I have learned three broad lessons from our experiments that may prove useful to industrial engineers. First, random mutation and selection can make a mediocre enzyme better, but don't often create entirely new activities [14]. The universe of possible proteins will never be explored. The average E. coli protein contains 360 amino acids so the number of possible proteins (composed of the standard 20 amino acids) of that length is $20^{360}=2 \times 10^{468}$. It is technically difficult to characterize a million cells at a time $\left(10^{6}=20^{4.6}\right)$. Populations of mutant proteins that differ in sequence from their ancestor by more than five mutations cannot be exhaustively characterized. Since five mutations is only $1.4 \%$ of the average protein, it is unlikely that entirely new activities could emerge from the population. I have nevertheless tried this experiment on several occasions, but so far without success. We currently believe that enzymes gain unselected secondary activities by chance [11], and that these novel functions are improved through Darwinian evolution in response to environmental change.

We also learned about the trade-offs between generalist and specialist enzymes. In macroscopic terms, generalists are species, such as the cockroach and rat, that consume a wide variety of foods. In contrast, specialists, like the giant panda and koala, excel at extracting energy from a single food source. Specialists thrive when the environment is stable, while generalists are more likely to survive periods of change. In our experience, however, directed enzyme evolution generally leads to enzyme variants that are broader in specificity (i.e. reactive with broader range of substrates) than the ancestral form (a naturally specific enzyme), even under conditions in which the environment is permanently changed from one state to another $[15,16]$. Specialist enzymes are apparently more architecturally elaborate, and therefore more difficult to evolve, than generalist enzymes.

Finally, we have come to appreciate the benefits of functional redundancy. Biological systems can fail, just as manufacturing equipment can break down, so the capacity of one enzyme to "pinch hit" for another enhances the robustness (resistance to perturbation) of the whole system. Redundancy also enhances evolvability, by enabling one copy to adapt to new circumstances, while another retains its original essential function [17]. The costs of redundancy upon biological systems and businesses are generally easier to estimate than the benefits. Furthermore, I expect that control theorists are biased toward simpler networks because they are easiest to model and explain. The emergent properties of more com-plicated complex systems are relatively difficult to predict, so it seems likely that industrial engineers disregard opportunities to make businesses more evolvable and more robust.

\section{Opportunities for Operations Researchers in Synthetic Biology and Metabolic Engineering}

Traditionally, biologists have conducted experiments to understand how living systems work. In recent years, however, some have begun to modify existing systems or to create completely artificial ones. The new discipline of synthetic biology was enabled by several new technologies. The cost of DNA sequencing continues to drop, so the number of possible "parts" for the design of biological "circuits" and "devices" continues to increase. The cost of gene synthesis also continues to decrease, so it is now possible to create new bacterial chromosomes that contain millions of nucleotide subunits [18]. Many DNA sequences are synthesized in accordance with the "BioBrick" standard [19], so that "parts" created in one lab can easily be combined with those from another. High throughput screens, like those employed for directed evolution experiments (vide supra), enable the trial-anderror of different designs on an industrial scale. Synthetic biologists have applied these tools to create bacterial cameras [20] and bacteria that swim toward theophylline, a natural drug derived from cocoa beans [21].

Some synthetic biologists assemble and express metabolic pathways in living cells, thereby converting them into microbial factories that add value to chemical feed stocks (metabolic engineering). The economic impact of any artificial biosynthetic pathway depends upon its yield. For example, biofuels get a lot of press (and venture capital funding) but all products of this fledgling industry are more expensive to produce than gasoline. New pathways generally require new regulatory mechanisms for optimal productivity. The host genome, that is the genes on the bacterial chromosome, can also be modified to improve production yields, but metabolic networks remain poorly characterized so it is difficult to know a priori how alterations in the flux of one pathway will affect the flux through another.

Metabolic engineers employ two complementary approaches. The rational design approach is exemplified by Bernhard Palsson and his co-workers, who formulated a computational model of the Escherichia coli metabolic network [22]. Metabolic engineers have used this model to guide the rational mutagenesis of the $E$. coli genome [23]. This approach, however, is limited by the mathematical accuracy of the model; most of the underlying parameters (i.e. the concentrations of enzymes metabolic 
intermediates, catalytic properties of hundreds of enzymes) have not been experimentally determined. Furthermore, the primary functions of many proteins, particularly those from poorly characterized organisms, remain unknown. The secondary activities described above remain mostly undocumented [11,12,24,25]. Metabolic network models are experimentally tested and refined by measuring overall constraints (growth rates, capacity to withstand mutation) [26]. In metabolic flux analyses, isotope-labeled substrates are fed to bacteria and tracked (by nuclear magnetic resonance or gas chromatographymass spectroscopy) as enzymes convert them into other compounds [23,27]. Functional genomics experiments will continue to elucidate the biological roles of uncharacterized Open Reading Frames (ORFs) [28].

Metabolic engineers also use random mutagenesis and screening to increase the flux through an economically valuable pathway. This classical approach mimics natural selection, but is generally labor-intensive and inefficient for two reasons. First, bacterial reproduction is generally asexual (no DNA recombination), which means that the evolving strains accumulate mildly deleterious mutations (Muller's Ratchet); beneficial alleles compete instead of recombine, and drive each other into extinction (clonal interference [29]). The second difficulty is the labor associated with high throughput screening. The desired secondary metabolite is rarely essential to the host organism, so the productivity of individual strains must be determined by techniques, such as automated reverse phase High Performance Liquid Chromatography (HPLC), that are relatively difficult to scale up in throughput.

Bacterial are "selfish", as they evolved to produce energy and chemical compounds for themselves rather than for human consumers. The efficient biosynthesis of pharmaceuticals, biofuels and biomaterials will likely require large scale restructuring of bacterial genomes. It is possible to direct the mutation of many genes [30,31]. One group has synthesized a whole bacterial chromosome, and has implanted it within a cell to demonstrate its functionality [18]. The capacity to change or create DNA, however, does not guarantee the design of more efficient processes. The latter will require an understanding of metabolic biochemistry and control theory. A few individuals possess expertise in both disciplines [23, 32-34] but many challenges remain so interdisciplinary collaborations are still likely to be productive.

\section{Conclusion}

Bacteria convert low value inputs into high value outputs, but unlike factories are products of evolution rather than intelligent design. In other words, bacteria and industrial engineers face similar problems but solve them in very different ways. Unfortunately, the biochemists who study metabolism and the operations researchers who study businesses generally work in different schools, speak different languages, and study their systems in very different ways. The traditional segregation of these disciplines creates some interesting collaborative opportunities. Can self-organizing systems improve the efficiencies of factories? Can evolutionary approaches be applied to the design of industrial processes? Can the mathematical models of operations research help explain the structure and organization of natural metabolic networks? Does control theory offer solutions to metabolic engineers? I hope that this essay provokes some interdisciplinary conversations that lead to the right questions.

\section{REFERENCES}

[1] R. Wolfenden, "Benchmark Reaction Rates, the Stability of Biological Molecules in Water, and the Evolution of Catalytic Power in Enzymes," Annual Review of Biochemistry, Vol. 80, No. 1, 2011, pp. 645-667. doi:10.1146/annurev-biochem-060409-093051

[2] A. E. Gray and J. Leonard, "Process Fundamentals," Harvard Business School, Boston, 2007.

[3] J. L. Goldstein and M. S. Brown, "Regulation of the Mevalonate Pathway,” Nature, Vol. 343, No. 6257, 1990, pp. 425-430. doi:10.1038/343425a0

[4] A. Koestler, "The Ghost in the Machine,” Arkana Penguin, Canada, 1989.

[5] R. C. Cadwell and G. F. Joyce, "Randomization of Genes by PCR Mutagenesis," PCR Methods and Applications, Vol. 2, No. 1, 1992, pp. 28-33. doi:10.1101/gr.2.1.28

[6] W. P. Stemmer, "Rapid Evolution of a Protein in Vitro by DNA Shuffling," Nature, Vol. 370, No. 6488, 1994, pp. 389-391. doi:10.1038/370389a0

[7] J. C. Moore and F. H. Arnold, "Directed Evolution of a Para-Nitrobenzyl Esterase for Aqueous-Organic Solvents," Nature Biotechnology, Vol. 14, No. 4, 1996, pp. 458-467. doi:10.1038/nbt0496-458

[8] W. Paley and J. Paxton, "Natural Theology: or, Evidences of the Existence and Attributes of the Deity, Collected from the Appearances of Nature," Gould and Lincoln, Lincoln, 1802.

[9] C. Darwin, "On the Origin of Species by Means of Natural Selection, or the Preservation of Favoured Races in the Struggle for Life,” John Murray, London, 1859.

[10] P. Vopalensky and Z. Kozmik, "Eye Evolution: Common Use and Independent Recruitment of Genetic Components," Philosophical Transactions of the Royal Society B: Biological Sciences, Vol. 364, No. 1531, 2009, pp. 28192832. doi:10.1098/rstb.2009.0079

[11] W. M. Patrick, et al., "Multicopy Suppression Underpins Metabolic Evolvability,” Molecular Biology and Evolution, Vol. 24, No. 12, 2007, pp. 2716-2722. doi:10.1093/molbev/msm204

[12] H. Nam, et al., "Network Context and Selection in the Evolution to Enzyme Specificity," Science, Vol. 337, No. 6098, 2012, pp. 1101-1104. doi:10.1126/science.1216861

[13] C. Zimmer, "Microcosm: E. coli and the New Science of 
Life,” Vintage, New York, 2009.

[14] I. Sarkar, et al., "HIV-1 Proviral DNA Excision Using an Evolved Recombinase,” Science, Vol. 316, No. 5833, 2007, pp. 1912-1915. doi:10.1126/science.1141453

[15] A. Aharoni, et al., "The 'Evolvability' of Promiscuous Protein Functions,” Nature Genetics, Vol. 37, No. 1, 2005, pp. 73-76.

[16] I. Matsumura and A. D. Ellington, "In Vitro Evolution of Beta-Glucuronidase into a Beta-Galactosidase Proceeds through Non-Specific Intermediates,” Journal of Molecular Biology, Vol. 305, No. 2, 2001, pp. 331-339. doi:10.1006/jmbi.2000.4259

[17] U. Bergthorsson, D. I. Andersson and J. R. Roth, “Ohno’s Dilemma: Evolution of New Genes under Continuous Selection," Proceedings of the National Academy of Sciences of the United States of America, Vol. 104, No. 43, 2007, pp. 17004-17009. doi:10.1073/pnas.0707158104

[18] D. G. Gibson, et al., "Creation of a Bacterial Cell Controlled by a Chemically Synthesized Genome," Science, Vol. 329, No. 5987, 2010, pp. 52-56. doi:10.1126/science.1190719

[19] R. P. Shetty, D. Endy and T. F. Knight Jr., "Engineering BioBrick Vectors from BioBrick Parts,” Journal of Biological Engineering, Vol. 2, No. 1, 2008, pp. 5-12 doi:10.1186/1754-1611-2-5

[20] A. Levskaya, et al., "Synthetic Biology: Engineering Escherichia coli to See Light,” Nature, Vol. 438, No. 7067, 2005, pp. 441-442. doi:10.1038/nature04405

[21] S. Topp and J. P. Gallivan, "Guiding Bacteria with Small Molecules and RNA," Journal of the American Chemical Society, Vol. 129, No. 21, 2007, pp. 6807-6811. doi:10.1021/ja0692480

[22] A. M. Feist, et al., "Reconstruction of Biochemical Networks in Microorganisms,” Nature Reviews Microbiology, Vol. 7, No. 2, 2009, pp. 129-143.

[23] S. K. Lee, et al., "Metabolic Engineering of Microorganisms for Biofuels Production: From Bugs to Synthetic Biology to Fuels,” Current Opinion in Biotechnology, Vol. 19, No. 6, 2008, pp. 556-563. doi:10.1016/j.copbio.2008.10.014

[24] S. Y. McLoughlin and S. D. Copley, "A Compromise Required by Gene Sharing Enables Survival: Implications for Evolution of New Enzyme Activities," Proceedings of the National Academy of Sciences of the United States of
America, Vol. 105, No. 36, 2008, pp. 13497-13502. doi:10.1073/pnas.0804804105

[25] W. M. Patrick and I. Matsumura, “A Study in Molecular Contingency: Glutamine Phosphoribosyl Pyrophosphate Amidotransferase is a Promiscuous and Evolvable Phosphoribosyl Anthranilate Isomerase," Journal of Molecular Biology, Vol. 377, No. 2, 2008, pp. 323-336. doi:10.1016/j.jmb.2008.01.043

[26] N. D. Price, J. L. Reed and B. O. Palsson, "Genome-Scale Models of Microbial Cells: Evaluating the Consequences of Constraints,” Nature Reviews Microbiology, Vol. 2, No. 11, 2004, pp. 886-897. doi:10.1038/nrmicro1023

[27] H. U. Kim, T. Y. Kim and S. Y. Lee, "Metabolic Flux Analysis and Metabolic Engineering of Microorganisms," Molecular BioSystems, Vol. 4, No. 2, 2008, pp. 113-120. doi:10.1039/b712395g

[28] H. Mori, et al., "Functional Genomics of Escherichia coli in Japan," Research in Microbiology, Vol. 151, No. 2, 2000, pp. 121-128. doi:10.1016/S0923-2508(00)00119-4

[29] P. J. Gerrish and R. E. Lenski, “The Fate of Competing Beneficial Mutations in an Asexual Population,” Genetica, Vol. 102-103, No. 1-6, 1998, pp. 127-144.

[30] H. H. Wang, et al., "Programming Cells by Multiplex Genome Engineering and Accelerated Evolution,” Nature, Vol. 460, No. 7257, 2009, pp. 894-898. doi:10.1038/nature08187

[31] J. R. Warner, et al., "Rapid Profiling of a Microbial Genome Using Mixtures of Barcoded Oligonucleotides," Nature Biotechnology, Vol. 28, No. 8, 2010, pp. 856-862. doi:10.1038/nbt.1653

[32] H. Alper, et al., "Identifying Gene Targets for the Metabolic Engineering of Lycopene Biosynthesis in Escherichia coli," Metabolic Engineering, Vol. 7, No. 3, 2005 , pp. 155-164. doi:10.1016/j.ymben.2004.12.003

[33] L. Kizer, et al., "Application of Functional Genomics to Pathway Optimization for Increased Isoprenoid Production," Applied and Environmental Microbiology, Vol. 74, No. 10, 2008, pp. 3229-3241. doi:10.1128/AEM.02750-07

[34] F. G. Vital-Lopez, et al., "A Computational Procedure for Optimal Engineering Interventions Using Kinetic Models of Metabolism,” Biotechnology Progress, Vol. 22, No. 6, 2006, pp. 1507-1517. 